\title{
Epidemiology and awareness of hypertension in a rural Ugandan community: a cross-sectional study
}

Prashant Kotwani ${ }^{1,2}$, Dalsone Kwarisiima ${ }^{3}$, Tamara D Clark ${ }^{1,2}$, Jane Kabami², Elvin H Geng ${ }^{1,2}$, Vivek Jain ${ }^{1,2}$, Gabriel Chamie ${ }^{1,2}$, Maya L Petersen ${ }^{4}$, Harsha Thirumurthy ${ }^{5}$, Moses R Kamya ${ }^{2,6}$, Edwin D Charlebois ${ }^{2,7}$, Diane $V$ Havlir ${ }^{1,2^{*}}$ and the SEARCH Collaboration

\begin{abstract}
Background: Hypertension is one of the largest causes of preventable morbidity and mortality worldwide. There are few population-based studies on hypertension epidemiology to guide public health strategies in sub-Saharan Africa. Using a community-based strategy that integrated screening for HIV and non-communicable diseases, we determined the prevalence, awareness, treatment rates, and sociodemographic factors associated with hypertension in rural Uganda.
\end{abstract}

Methods: A household census was performed to enumerate the population in Kakyerere parish in Mbarara district, Uganda. A multi-disease community-based screening campaign for hypertension, diabetes, and HIV was then conducted. During the campaign, all adults received a blood pressure (BP) measurement and completed a survey examining sociodemographic factors. Hypertension was defined as elevated BP $(\geq 140 / \geq 90 \mathrm{mmHg})$ on the lowest of three BP measurements or current use of antihypertensives. Prevalence was calculated and standardized to age distribution. Sociodemographic factors associated with hypertension were evaluated using a log-link Poisson regression model with robust standard errors.

Results: Community participation in the screening campaign was 65\%, including 1245 women and 1007 men. The prevalence of hypertension was 14.6\%; awareness of diagnosis (38.1\%) and current receipt of treatment (20.6\%) were both low. Age-standardized to the WHO world standard population, hypertension prevalence was 19.8\%, which is comparable to $21.6 \%$ in the US and $18.4 \%$ in the UK. Sociodemographic factors associated with hypertension included increasing age, male gender, overweight, obesity, diabetes, alcohol consumption, and family history. Prevalence of modifiable factors was high: $28.3 \%$ women were overweight/obese and $24.1 \%$ men consumed $\geq 10$ alcoholic drinks per month.

Conclusions: We found a substantial burden of hypertension in rural Uganda. Awareness and treatment of hypertension is low in this region. Enhanced community-based education and prevention efforts tailored to addressing modifiable factors are needed.

Keywords: Hypertension, Epidemiology, Blood pressure, Non-communicable disease, Community health, Health campaign, Public health, Sub-Saharan Africa, Uganda, Rural

\footnotetext{
* Correspondence: dhavlir@php.ucsf.edu

'HIV/AIDS Division, Department of Medicine, San Francisco General Hospital,

University of California San Francisco, 995 Potrero Avenue, UCSF Box 0874,

San Francisco, California 94110, USA

${ }^{2}$ Makerere University-University of California San Francisco (MU-UCSF)

Research Collaboration, Mbarara, Uganda

Full list of author information is available at the end of the article
} 


\section{Background}

Increasing urbanization has fueled social and economic changes in sub-Saharan Africa, which have contributed to a surge in non-communicable disease (NCD), including hypertension [1,2]. High blood pressure is the leading risk factor for mortality worldwide and approximately $80 \%$ of all cardiovascular deaths occur in lowand middle-income countries [3-5]. It is predicted that more than 125 million adults in sub-Saharan Africa alone will have hypertension by 2025 [6,7]. Given the long-term decreased productivity associated with hypertension, identifying and treating a large proportion of patients has the potential to generate tremendous social and economic benefits in this region [8-13].

Despite the recognition of hypertension as a major threat to public health in sub-Saharan Africa, epidemiologic research to guide public health approaches to prevent and treat hypertension is limited in scope, sample size, methodology, and generalizability of conclusions [7,8,12,14-16]. Robust population-based data using sound statistics are still needed to confirm prior estimates and inform policy debates. Further, strategies that allow hypertension screening on a population level need to be developed in these resource-limited settings.

Novel community- and home-based testing programs are being deployed as a means to diagnose and treat HIV in sub-Saharan Africa $[17,18]$. These programs address barriers such as high cost of accessing clinic-based testing, inadequate primary care services and lack of awareness about testing. Leveraging this HIV targeted infrastructure can improve services for NCDs, especially in the context of a fragile health care delivery system [12,19-22]. Building these strategic partnerships can also be used to study the epidemiology of NCDs.

The Sustainable East Africa Research in Community Health (SEARCH) collaboration has pioneered multidisease prevention and treatment services that integrate HIV and NCD services through community-based screening campaigns. In a prior pilot campaign conducted in May 2011 in a rural community of southwestern Uganda, we found a significant burden of untreated hypertension [23]. In the present study, we sought to determine the prevalence, awareness, treatment rates and sociodemographic factors associated with hypertension in this community using an approach that integrates HIV and NCD testing.

\section{Methods}

\section{Study setting}

The study was conducted in Kakyerere parish within Mbarara district in southwestern Uganda. A parish is an administrative unit in Uganda that consists of multiple villages. Kakyerere parish includes 9 villages comprising approximately 6,500 persons with $50 \%$ of the population $\geq 18$ years [23].

\section{Community census}

A community-wide household level census was performed in Kakyerere parish. Trained staff, with the help of local leaders, identified and attempted to visit all households within the boundary of the parish. When a visit was made, the head of the household provided informed consent to collect demographic information about all household members. A unique householdidentifier was given to each household, which was used to accurately identify participants at the community health campaign. The census allowed ascertainment of community participation in the campaign, understanding of basic sociodemographics of non-participants, and calculation of age-standardized measures for the whole parish population.

\section{Community health campaign}

Community health campaigns $(\mathrm{CHC})$ are large public health initiatives that provide rapid diagnostic services for multiple diseases in rural areas. Prior to the campaign, local leaders designed and executed community mobilization measures including radio announcements and posters in order to maximize participation at the $\mathrm{CHC}$. Local health personnel staffed each campaign and provided services for adults and children at community sites such as schools and churches. The $\mathrm{CHC}$ occurred over five days and each adult participant received pointof care screening for HIV, hypertension, and diabetes. Those screening positive for any disease were given a follow-up appointment at a local health facility for further management. Children were offered HIV testing, rapid diagnosis and treatment for malaria, and deworming. Each person who joined in the $\mathrm{CHC}$ participated on one campaign day for 2-3 hours. The campaign is "high throughput" because it provides rapid services for a large population (approximately 1,000 persons/day). Detailed procedures regarding the implementation of a $\mathrm{CHC}$ in Kakyerere parish have been recently described by our group [23].

\section{Blood pressure measurement}

All adult $\mathrm{CHC}$ participants received blood pressure (BP) screening and completed an epidemiologic survey at the CHC. Inclusion criteria were age $\geq 18$ years and residence in Kakyerere parish. Hypertension was defined as systolic BP $\geq 140 \mathrm{mmHg}$ or diastolic $\mathrm{BP} \geq 90 \mathrm{mmHg}$ per the World Health Organization (WHO)/International Society of Hypertension (ISH) guidelines [24]. Hypertension was further classified as Stage 1 (140-159/90-99 $\mathrm{mmHg}$ ) or Stage $2(\geq 160 / \geq 100 \mathrm{mmHg})$ based on the Seventh report of the Joint National Committee on prevention, 
detection, evaluation, and treatment of high blood pressure (JNC7) guidelines [25].

Trained staff conducted BP measurements using an electronic, automated sphygmomanometer (Honsun LD7A), with small $(<21 \mathrm{~cm})$, normal $(22-32 \mathrm{~cm})$, and large $(>33 \mathrm{~cm})$ cuff sizes. Subjects were seated in a chair and rested for approximately one minute before $\mathrm{BP}$ was measured. Following initial BP measurement, participants who met criteria for hypertension during this screen underwent two repeat measurements one minute apart. The lowest of three measurements was used to establish a diagnosis of hypertension.

Height was measured to the nearest $0.1 \mathrm{~cm}$ after removal of shoes. Weight was measured to the nearest kilogram after removal of shoes and heavy clothing. Body mass index (BMI) was calculated to determine proportion of overweight $\left(25.0-29.9 \mathrm{~kg} / \mathrm{m}^{2}\right)$ and obese $\left(\geq 30 \mathrm{~kg} / \mathrm{m}^{2}\right)$ participants. In addition, point-of-care random plasma glucose (Optium Xeed - Abbot) and HIV rapid tests (Determine HIV-1/HIV-2 (Abbot); HIV-1/2 Stat-Pak assay; and Uni-Gold HIV Rapid test) were also performed. Trained staff conducted a survey to determine sociodemographic factors and treatment history for hypertension.

\section{Hypertension treatment referral}

Hypertensive adults identified at the $\mathrm{CHC}$ were given a referral appointment to a local government health facility, where they received treatment per the Ugandan Ministry of Health guidelines [26]. Government health facilities in Uganda are required to offer free services to all persons, including supply of antihypertensive medications. In the event of antihypertensive drug shortages at government facilities, patients are occasionally referred to private retail pharmacies to obtain antihypertensive medications. In a previous report, the monthly cost of purchasing antihypertensives has been estimated at 1.1 Euros (1.4 US Dollars) in a similar region of sub-Saharan Africa [27].

\section{Data analysis}

Survey data were collected via a computerized interview programmed onto data tablets. Stata v.12 (Stata Corporation) was used for all analyses.

Hypertension was defined as systolic BP $\geq 140 \mathrm{mmHg}$ or diastolic BP $\geq 90 \mathrm{mmHg}$ on lowest of three BP measures or current use of antihypertensives. Age-standardized prevalence of hypertension was calculated using the Kakyerere parish community-wide census population, 2000 US census population [28], and WHO world standard population [29]. Awareness of a diagnosis of hypertension was defined by self-report among those determined to be hypertensive. BP control was defined as having $\mathrm{BP}$ below hypertension threshold (systolic
$\mathrm{BP}<140$ and diastolic $\mathrm{BP}<90 \mathrm{mmHg}$ ) if currently taking antihypertensives.

We conducted a sensitivity analysis on our prevalence estimate to account for transient changes in BP levels. The upper estimate was calculated by including those with self-reported hypertension but normal BP on screening. The lower estimate was calculated by using a stricter definition of hypertension, systolic BP $\geq 160$ or diastolic BP $\geq 95 \mathrm{~mm} \mathrm{Hg}$.

Sociodemographic factors that may be associated with hypertension prevalence were analyzed. Specifically, we evaluated age, gender, BMI, level of education, occupation, smoking status, alcohol consumption, family history, and random plasma glucose. Due to well-established links between HIV and cardiovascular complications [30], we also assessed HIV status.

Associations between sociodemographic factors and hypertension were investigated using a multivariable loglink Poisson model with robust standard errors to estimate adjusted prevalence ratios. All sociodemographic factors were included a priori in the multivariable regression model. Analyses were stratified by gender.

\section{Ethics}

The study was reviewed and approved by the Makerere University School of Medicine Research and Ethics Committee, the Ugandan National Council on Science and Technology, and the UCSF Committee on Human Research.

\section{Results}

\section{Characteristics of study participants}

2,282 adults attended the CHC and 2,252 (99\%) were screened for hypertension and answered epidemiologic survey at the CHC. These persons represented $65 \%$ of the 3,485 adults (1,861 women and 1,624 men) enumerated in the Kakyerere parish community census. Participation in the $\mathrm{CHC}$ was higher among women (66.9\%) than men $(62.0 \%)(\mathrm{P}=0.002)$ (Table 1). Participation was lowest among 18-29 year olds for both women (56.6\%, $\mathrm{P}<0.0001)$ and men $(58.4 \%, \mathrm{P}=0.005)$.

Demographic and clinical characteristics are listed in Table 2.

\section{Prevalence, awareness, treatment and control of hypertension}

The overall unadjusted prevalence of hypertension in this community was $15.7 \%$. Hypertension prevalence was $16.1 \%$ among men and $15.4 \%$ in women $(\mathrm{P}=0.67)$ (Table 2). Per JNC7 classification [25], 9.4\% participants had Stage 1 hypertension while $5.0 \%$ had Stage 2 hypertension.

A large fraction of patients were not aware of being hypertensive and received a new diagnosis at the $\mathrm{CHC}$. 
Table 1 Number of community members screened for hypertension at the community health campaign (CHC) in Kakyerere parish, in categories of age and gender

\begin{tabular}{ccc}
\hline Women: & & \\
Age & Persons screened & Community participation (\%) \\
$18-29$ & 478 & 56.6 \\
$30-44$ & 415 & 75.4 \\
$45-59$ & 194 & 75.8 \\
$\geq 60$ & 158 & 75.2 \\
Total & 1245 & 66.9 \\
Men: & & \\
Age & Persons screened & Community participation (\%) \\
$18-29$ & 448 & 58.4 \\
$30-44$ & 315 & 62.4 \\
$45-59$ & 151 & 68.9 \\
$\geq 60$ & 93 & 69.4 \\
Total & 1007 & 62.0 \\
\hline
\end{tabular}

Awareness was higher among women: 50.5\% of hypertensive women were aware of their diagnosis compared to only $23.5 \%$ men $(P<0.0001)$. Self reported use of antihypertensives was also significantly more common among women $(28.6 \%)$ compared to men $(11.1 \%) \quad(\mathrm{P}<0.0001)$. Among those using antihypertensive medication, a similar proportion of women $(40.0 \%)$ and men (38.9\%) achieved $\mathrm{BP}$ control (normotensive on reported therapy) $(\mathrm{P}=0.93)$.

\section{Age-standardized prevalence}

Using the Kakyerere parish household census population age distribution, the age-standardized prevalence for the entire community was $14.6 \%$, (95\% confidence interval (CI) 13.3-15.9; with women at $13.4 \%$, and men at $16.0 \%$ ). Using the 2000 US census population and the WHO world standard population, the age-standardized prevalence was $22.0 \%$ (95\% CI 20.0-24.0) and 19.8\% (95\% CI 18.0-21.5), respectively (Table 3 ).

\section{Sociodemographic factors associated with hypertension}

Among women, significant associations with hypertension in the adjusted regression model included age, BMI, and diabetes (random plasma glucose $\geq 11.1 \mathrm{mmol} / \mathrm{L}$ ) (Table 4). Age $\geq 60$ years was associated with a marked elevation in hypertension prevalence compared to age $18-29$ years $(P R=12.30 ; 95 \%$ CI $6.59-22.10)$. Similarly, the prevalence of hypertension increased with BMI: overweight and obese women were more likely to be hypertensive $(\mathrm{PR}=1.75 ; 95 \% \mathrm{CI} 1.28-2.40$, and $\mathrm{PR}=2.72$; 95\% CI 1.94-3.83, respectively). Diabetes was also associated with substantially increased likelihood of being hypertensive $(\mathrm{PR}=2.44 ; 95 \% \mathrm{CI} 1.32-4.51)$.
Among men, sociodemographic predictors of hypertension included age, BMI, alcohol use, HIV status, and family history. Increasing age was associated with an increase in the prevalence of hypertension: older men ( $\geq 60$ years) were six times as likely to have hypertension compared to younger men (18-29 years), PR $=6.00$ (95\% CI 3.10-11.58). Increase in BMI was also associated with a greater hypertension prevalence: $\mathrm{PR}=2.12(95 \% \mathrm{CI}$ 1.45-3.11) for overweight men and $\mathrm{PR}=1.64$ (95\% CI 0.84-3.22) for obese men. Men consuming $\geq 10$ alcoholic drinks per month had $60 \%$ higher prevalence of hypertension compared to non-drinkers, $\mathrm{PR}=1.60(95 \% \mathrm{CI}$ 1.13-2.26). HIV infected men were less likely to be hypertensive, $\mathrm{PR}=0.39$ (95\% CI $0.17-0.87$ ). Men with a family history of hypertension in first degree relatives were 1.5 times as likely to have hypertension than those without a family history, PR $=1.51$ (95\% CI 1.12-2.04).

In a separate multivariable model including gender, men were 1.3 times more likely to have hypertension than women after adjustment for all other variables, PR $=1.34$ (95\% CI 1.05-1.69) (data not shown).

\section{Sensitivity analysis}

Estimated prevalence of hypertension was 19.7\% (women $20.8 \%$ and men 18.3\%) if those with self-reported hypertension were considered. A lower estimate of $6.6 \%$ (women $6.7 \%$ and men 6.4\%) was obtained by using more stringent $\mathrm{BP}$ measures (SBP $\geq 160$ or $\mathrm{DBP} \geq 95 \mathrm{~mm} \mathrm{Hg}$ ).

\section{Discussion}

Using a community-based strategy that integrated HIV and NCD screening, we found a substantial burden of hypertension (14.6\% age-standardized prevalence) accompanied by low rates of awareness of the condition. Among individuals aware of their hypertension, we found low rates of therapy. Furthermore, we found a high prevalence of multiple modifiable factors associated with hypertension. Our study, by leveraging a multidisease $\mathrm{CHC}$ to study hypertension, demonstrates that strategic public health investments in HIV and NCDs can be complementary and efficient in providing diagnostic services in Sub-Saharan Africa.

Using this community-based approach we provide estimates of hypertension prevalence in the range of those previously reported from Uganda [12,31] and sub-Saharan Africa [7]. Age-standardized to the WHO world standard population, hypertension prevalence in Kakyerere parish was $19.8 \%$, which is comparable to $21.6 \%$ in the US and $18.4 \%$ in the UK [32]. This finding adds to accumulating evidence that prevalence of hypertension in sub-Saharan Africa is approaching that in developed nations [6,32-35]. Further, our data are especially concerning when considering that rural areas in Sub-Saharan Africa have a lower prevalence 
Table 2 Demographic and clinical characteristics of study participants

$\begin{array}{ccc}\text { Women } & \text { Men } & \text { Total } \\ (\mathrm{N}=1245) & (\mathrm{N}=1007) & (\mathrm{N}=2252) \\ & \mathrm{N}(\%) & \end{array}$

\section{Age (years)}

$18-29$

$478(38.4 \%)$

$30-44$

$415(33.3 \%)$

$448(44.5 \%)$

$926(41.1 \%)$

$45-59$

$194(15.6 \%)$

315 (31.3\%)

730 (32.4\%)

$\geq 60$

$158(12.7 \%)$

151 (15.0\%)

$345(15.3 \%)$

Marital status ${ }^{\mathrm{a}}$

Single

$172(14.6 \%)$

$93(9.2 \%)$

$251(11.2 \%)$

Married (ever)

$1009(85.4 \%)$

300 (32.7\%)

$472(22.5 \%)$

BMI $\left(\mathrm{kg} / \mathrm{m}^{2}\right)^{\mathrm{b}}$

$\leq 25$

$892(71.7 \%)$

$25-30$

$243(19.5 \%)$

$618(67.3 \%)$

$1627(77.5 \%)$

$\geq 30$

$110(8.8 \%)$

$923(91.7 \%)$

1815 (80.6\%)

$68(6.8 \%)$

311 (13.8\%)

\section{Occupation}

Unemployed

$158(12.7 \%)$

Manual

$1014(81.4 \%)$

$15(1.5 \%)$

$125(5.5 \%)$

Sedentary

73 (5.9\%)

176 (17.5\%)

334 (14.8\%)

$1772(78.7 \%)$

$146(6.5 \%)$

\section{Education level}

No school

$310(24.9 \%)$

Primary

$651(52.3 \%)$

758 (75.3\%)

Secondary

Tertiary and beyond

$222(17.8 \%)$

$62(5.0 \%)$

Acres of land owned ${ }^{c}$

None

$142(12.4 \%)$

$282(24.5 \%)$

484 (42.1\%)

$242(21.0 \%)$

94 (9.3\%)

404 (17.9\%)

$532(52.8 \%)$

$1183(52.5 \%)$

519 (23.1\%)

297 (29.5\%)

$146(6.5 \%)$

$\leq 1$

1-5

$\geq 5$

1208 (97.0\%)

783 (77.8\%)

991 (88.4\%)

37 (3.0\%)

$224(22.2 \%)$

261 (11.6\%)

Alcohol use (drinks consumed per month) ${ }^{d}$

None

986 (79.3\%)

$527(52.4 \%)$

1513 (67.3\%)

196 (15.7\%)

$236(23.5 \%)$

432 (19.2\%)

$\geq 10$

62 (5.0\%)

242 (24.1\%)

304 (13.5\%)

HIV

Negative

1107 (88.9\%)

$923(91.7 \%)$

2030 (90.1\%)

Positive

138 (11.1\%)

84 (8.3\%)

222 (9.9\%)

Random plasma glucose (mmol/L)

0-7

1101 (88.7\%)

895 (89.2\%)

1996 (88.9\%)

7-11

126 (10.2\%)

103 (10.3\%)

229 (10.2\%)

$\geq 11.1$

$5(0.5 \%)$

19 (0.9\%) 
Table 2 Demographic and clinical characteristics of study participants (Continued)

\begin{tabular}{|c|c|c|c|}
\hline \multicolumn{4}{|l|}{ Family history $^{f}$} \\
\hline No & $962(79.5 \%)$ & $798(81.6 \%)$ & $1760(80.4 \%)$ \\
\hline Yes & $248(20.5 \%)$ & $180(18.4 \%)$ & $428(19.6 \%)$ \\
\hline \multicolumn{4}{|l|}{ Blood pressure (BP) (mm Hg) } \\
\hline Systolic Blood Pressure Mean (SD) & $122.5(18.6)$ & $125.5(16.7)$ & $123.8(17.9)$ \\
\hline Diastolic Blood Pressure Mean (SD) & $74.9(12.1)$ & $74.8(11.7)$ & $74.9(11.9)$ \\
\hline Hypertension (elevated BP or taking antihypertensives) & $192(15.4 \%)$ & $162(16.1 \%)$ & $354(15.7 \%)$ \\
\hline $\mathrm{BP} \geq 140 / \geq 90 \mathrm{~mm} \mathrm{Hg}$ & $170(13.7 \%)$ & $155(15.4 \%)$ & $325(14.4 \%)$ \\
\hline Stage 1 (140-159/90-99) & $104(8.4 \%)$ & $108(10.7 \%)$ & $212(9.4 \%)$ \\
\hline Stage $2(\geq 160 / \geq 100)$ & $66(5.3 \%)$ & $47(4.7 \%)$ & $113(5.0 \%)$ \\
\hline Self-reported hypertension & $164(13.2 \%)$ & $60(6.0 \%)$ & $224(10.0 \%)$ \\
\hline Awareness (if hypertensive, $\mathrm{N}=354$ ) & $97(50.5 \%)$ & $38(23.5 \%)$ & $135(38.1 \%)$ \\
\hline Receiving treatment (if hypertensive, $\mathrm{N}=354$ ) & $55(28.6 \%)$ & $18(11.1 \%)$ & $73(20.6 \%)$ \\
\hline $\mathrm{BP}$ controlled (if receiving treatment, $\mathrm{N}=73$ ) & $22(40.0 \%)$ & 7 (38.9\%) & $29(39.7 \%)$ \\
\hline
\end{tabular}

${ }^{\mathrm{a}} \mathrm{N}=2099$, female $=1181$, male $=918$.

${ }^{b} \mathrm{~N}=2251$, female $=1245$, male $=1006$.

${ }^{\mathrm{C}} \mathrm{N}=2095$, female $=1150$, male $=945$.

${ }^{\mathrm{d}} \mathrm{N}=2249$, female $=1244$, male $=1005$.

$e^{e} \mathrm{~N}=2244$, female $=1241$, male $=1003$.

${ }^{f} \mathrm{~N}=2188$, female $=1210$, male $=978$.

of hypertension compared to semi-urban and urban areas [16,36-39].

Awareness of a hypertension diagnosis (38.1\%) was inadequate and especially poor among men (23.5\%). In a review, Addo et al. [11] report that hypertension awareness in sub-Saharan Africa was generally below 40\%, consistent with our results. Despite being aware, however, only one out of five known hypertensives was receiving treatment. Further, BP control was achieved in only $39.7 \%$ of hypertensive patients on therapy. Addressing these gaps requires health system strengthening. Community health campaigns can be a key component of new strategies that build both individual and community level awareness of NCDs such as hypertension.

Sociodemographic factor analysis identified groups of persons most likely to be hypertensive - these persons can serve as the target for prevention strategies in rural Uganda. Further, modifiable factors associated with hypertension in this population include overweight, obesity, alcohol consumption, and diabetes-all consistent with prior reports $[9,12,15,31,40]$. BMI and hypertension had a strong relationship among both women and men, while a relationship with alcohol consumption $(\geq 10$ drinks/month) was found in men only. Three out of ten women were either overweight or obese compared to only one out of ten men. $24.1 \%$ men consumed $\geq 10$ alcoholic drinks/month compared to only $5 \%$ women.

Our data also emphasize the need for greater diagnosis and treatment of diabetes, particularly among women. The small number of men found to have elevated blood glucose prevented meaningful analysis of the link between diabetes and hypertension in men. Consistent with other studies across sub-Saharan Africa [11,12,15,40], hypertension had a strong relationship with both increasing age and male gender. Family history was independently related to hypertension among men but not women. These data can be utilized to stratify risk and inform hypertension treatment decisions in rural Uganda.

Interestingly, HIV infected men were less likely to be hypertensive even after multivariable adjustment, $\mathrm{PR}=0.39$ (0.17-0.87). Previously, Barnighausen et al. reported a $3.0 \mathrm{~mm} \mathrm{Hg}$ decrease in systolic BP with

Table 3 Age-standardized prevalence of hypertension using the Kakyerere Parish household census population, the 2000 U.S. standard population, and the WHO world standard population among adults $\geq 18$ years

\begin{tabular}{lccc}
\hline & \multicolumn{2}{c}{ Prevalence (95\% CI) } \\
\cline { 2 - 4 } & $\begin{array}{c}\text { Study participants } \\
\text { (unadjusted) }\end{array}$ & $\begin{array}{c}\text { Kakyerere Parish household } \\
\text { census population }\end{array}$ & $\begin{array}{c}\text { 2000 US census } \\
\text { population }\end{array}$ \\
\hline Total & $15.7 \%(14.2-17.2)$ & $14.6 \%(13.3-15.9)$ & $22.0 \%(20.0-24.0)$ \\
Women & $15.4 \%(13.4-17.4)$ & $13.4 \%(11.7-15.1)$ & $21.1 \%(18.5-23.6)$ \\
Men & $16.1 \%(13.8-18.4)$ & $16.0 \%(13.9-18.2)$ & $23.1 \%(19.9-26.2)$ \\
\hline
\end{tabular}


Table 4 Risk factors associated with hypertension using a log-link Poisson model with robust standard errors

\begin{tabular}{|c|c|c|c|c|c|c|}
\hline & & Nomen $\left(\mathrm{N}=1245^{\mathrm{a}}\right)$ & & & Men $\left(\mathrm{N}=1007^{\mathrm{a}}\right)$ & \\
\hline & $\begin{array}{l}\text { Proportion with } \\
\text { hypertension (\%) }\end{array}$ & $\begin{array}{c}\text { Unadjusted } \\
\text { prevalence ratio } \\
\text { PR }(95 \% \mathrm{Cl})\end{array}$ & $\begin{array}{c}\text { Adjusted }^{\mathbf{b}} \\
\text { prevalence ratio } \\
\text { PR }(95 \% \mathrm{Cl})\end{array}$ & $\begin{array}{c}\text { Proportion with } \\
\text { hypertension } \\
(\%)\end{array}$ & $\begin{array}{c}\text { Unadjusted } \\
\text { prevalence ratio } \\
\text { PR }(95 \% \mathrm{Cl})\end{array}$ & $\begin{array}{c}\text { Adjusted }^{\mathbf{b}} \\
\text { prevalence ratio } \\
\text { PR }(95 \% \mathrm{Cl}) \\
\end{array}$ \\
\hline Age (years) & & $P<0.0001$ & $P<0.0001$ & & $P<0.0001$ & $P<0.0001$ \\
\hline $18-29$ & $16 / 478=3.4 \%$ & 1 & 1 & $29 / 326=6.5 \%$ & 1 & 1 \\
\hline $30-44$ & $58 / 415=14.0 \%$ & $4.18(2.44-7.15)$ & $3.93(2.15-7.18)$ & $50 / 315=15.9 \%$ & $2.45(1.59-3.79)$ & $2.13(1.10-4.11)$ \\
\hline $45-59$ & $48 / 194=24.7 \%$ & $7.39(4.30-12.70)$ & $6.20(3.33-11.55)$ & $44 / 151=29.1 \%$ & $4.50(2.92-6.93)$ & $3.94(2.05-7.56)$ \\
\hline$\geq 60$ & $70 / 158=44.3 \%$ & $13.24(7.93-22.10)$ & 12.30 (6.59-22.96) & $39 / 93=41.9 \%$ & 6.48 (4.23-9.92) & $6.00(3.10-11.58)$ \\
\hline Marital Status & & $P=0.006$ & $P=0.35$ & & $P<0.0001$ & $P=0.94$ \\
\hline Single & $14 / 172=8.1 \%$ & 1 & 1 & $23 / 300=7.7 \%$ & 1 & 1 \\
\hline Married (ever) & $171 / 1009=16.9 \%$ & $2.08(1.24-3.50)$ & $0.79(0.48-1.30)$ & $132 / 618=21.4 \%$ & $2.79(1.83-4.24)$ & $1.03(0.53-2.01)$ \\
\hline BMI $\left(\mathrm{kg} / \mathrm{m}^{2}\right)$ & & $P<0.0001$ & $P<0.0001$ & & $P<0.0001$ & $P=0.0004$ \\
\hline$<25$ & $105 / 892=11.8 \%$ & 1 & 1 & $130 / 923=14.1 \%$ & 1 & 1 \\
\hline $25-30$ & $48 / 243=19.7 \%$ & $1.68(1.23-2.29)$ & $1.75(1.28-2.40)$ & $24 / 68=35.3 \%$ & $2.51(1.75-3.59)$ & $2.12(1.45-3.11)$ \\
\hline$>30$ & $39 / 110=35.5 \%$ & $3.01(2.21-4.1)$ & $2.72(1.94-3.83)$ & $7 / 15=46.7 \%$ & $3.31(1.89-5.83)$ & $1.64(0.84-3.22)$ \\
\hline Occupation & & $P=0.99$ & $P=0.36$ & & $P=0.06$ & $P=0.92$ \\
\hline Unemployed & $24 / 158=15.2 \%$ & 1 & 1 & $20 / 176=11.4 \%$ & 1 & 1 \\
\hline Manual & $157 / 1014=15.5 \%$ & $1.02(0.69-1.51)$ & $0.79(0.53-1.18)$ & $125 / 758=16.5 \%$ & $1.45(0.93-2.26)$ & $1.06(0.67-1.69)$ \\
\hline Sedentary & $11 / 73=15.1$ & $0.99(0.51-1.92)$ & $1.09(0.57-2.10)$ & $17 / 73=23.3 \%$ & $2.05(1.14-3.68)$ & $1.16(0.57-2.35)$ \\
\hline Education & & $P<0.0001$ & $P=0.61$ & & $P=0.05$ & $P=0.43$ \\
\hline No school & $80 / 310=25.8 \%$ & 2.05 (1.55-2.70) & $1.19(0.88-1.62)$ & $19 / 94=20.2 \%$ & $1.25(0.80-1.95)$ & $1.15(0.73-1.80)$ \\
\hline Primary & $82 / 651=12.6 \%$ & 1 & 1 & $86 / 532=16.2 \%$ & 1 & 1 \\
\hline Secondary & $23 / 222=10.4 \%$ & $0.82(0.53-1.27)$ & $1.22(0.77-1.94)$ & $37 / 297=12.5 \%$ & $0.77(0.54-1.10)$ & $1.03(0.70-1.50)$ \\
\hline Tertiary and beyond & $7 / 62=11.3 \%$ & $0.90(0.43-1.85)$ & $1.00(0.44-2.28)$ & $20 / 84=23.8 \%$ & $1.47(0.96-2.26)$ & $1.52(0.90-2.57)$ \\
\hline Acres of land owned & & $P=0.50$ & $P=0.56$ & & $P=0.58$ & $P=0.90$ \\
\hline None & $17 / 142=12.0 \%$ & 1 & 1 & $11 / 96=11.5 \%$ & 1 & 1 \\
\hline$\leq 1$ & $50 / 282=17.7 \%$ & $1.48(0.89-2.47)$ & $1.47(0.86-2.52)$ & $27 / 157=17.2 \%$ & $1.50(0.78-2.88)$ & $1.03(0.54-1.98)$ \\
\hline $1-5$ & $76 / 484=15.7 \%$ & $1.31(0.80-2.14)$ & $1.30(0.78-2.18)$ & $64 / 370=17.3 \%$ & $1.51(0.83-2.75)$ & $1.17(0.65-2.09)$ \\
\hline$\geq 5$ & $40 / 242=16.5 \%$ & $1.38(0.81-2.34)$ & $1.24(0.72-2.14)$ & $51 / 322=15.8 \%$ & $1.38(0.75-2.54)$ & $1.07(0.59-1.93)$ \\
\hline Tobacco use & & $P=0.54$ & $P=0.49$ & & $P=0.22$ & $P=0.70$ \\
\hline No & $185 / 1208=15.3 \%$ & 1 & 1 & $120 / 783=15.3 \%$ & 1 & 1 \\
\hline Yes & $7 / 37=18.9 \%$ & $1.24(0.63-2.44)$ & $0.78(0.39-1.57)$ & $42 / 224=18.8 \%$ & $1.22(0.89-1.68)$ & $0.94(0.67-1.31)$ \\
\hline Alcohol use & & $P=0.70$ & $P=0.33$ & & $P<0.0001$ & $P=0.02$ \\
\hline None & $148 / 986=15.0 \%$ & 1 & 1 & $59 / 527=11.2 \%$ & 1 & 1 \\
\hline $0-10$ & $34 / 196=17.4 \%$ & $1.16(0.82-1.62)$ & $0.92(0.66-1.28)$ & $38 / 236=16.1 \%$ & $1.44(0.99-2.10)$ & $1.17(0.79-1.71)$ \\
\hline$\geq 10$ & $10 / 62=16.1 \%$ & $1.07(0.60-1.93)$ & $0.58(0.28-1.21)$ & $64 / 242=26.5 \%$ & $2.36(1.72-3.25)$ & $1.60(1.13-2.26)$ \\
\hline HIV & & $P=0.005$ & $P=0.24$ & & $P=0.10$ & $P=0.02$ \\
\hline Negative & $183 / 1107=16.5 \%$ & 1 & 1 & $154 / 923=16.7 \%$ & 1 & 1 \\
\hline Positive & $9 / 138=6.5 \%$ & $0.39(0.21-0.75)$ & $0.69(0.37-1.28)$ & $8 / 84=9.5 \%$ & $0.57(0.29-1.12)$ & $0.39(0.17-0.87)$ \\
\hline$R P G^{d}$ & & $P<0.0001$ & $P=0.02$ & & $P=0.0002$ & $P=0.75$ \\
\hline$<7$ & $160 / 1101=14.5 \%$ & 1 & 1 & $135 / 895=15.1 \%$ & 1 & 1 \\
\hline $7-11$ & $23 / 126=18.3 \%$ & $1.26(0.84-1.87)$ & $1.02(0.68-1.52)$ & $24 / 103=23.3 \%$ & $1.54(1.05-2.27)$ & $1.17(0.78-1.76)$ \\
\hline$\geq 11.1$ & $8 / 14=57.1 \%$ & $3.93(2.44-6.33)$ & $2.44(1.32-4.51)$ & $3 / 5=60.0 \%$ & $3.98(1.91-8.28)$ & $1.10(0.58-2.09)$ \\
\hline
\end{tabular}


Table 4 Risk factors associated with hypertension using a log-link Poisson model with robust standard errors (Continued)

\begin{tabular}{lcccccc}
\hline Family history & & $P=0.11$ & $P=0.11$ & & $P=0.002$ & $P=0.007$ \\
No & $139 / 962=14.5 \%$ & 1 & 1 & $114 / 798=14.3 \%$ & 1 & 1 \\
Yes & $46 / 248=18.6 \%$ & $1.28(0.95-1.74)$ & $1.26(0.95-1.69)$ & $42 / 180=23.3 \%$ & $1.63(1.19-2.24)$ & $1.51(1.12-2.04)$ \\
\hline
\end{tabular}

${ }^{\mathrm{a}} 1067$ women and 839 men included in the multivariate regression model.

${ }^{b}$ Each factor is adjusted for all other factors in the table.

'Drinks of alcohol consumed per month.

${ }^{\mathrm{d}} \mathrm{RPG}$ - Random plasma glucose in $\mathrm{mmol} / \mathrm{L}$.

HIV infection after adjusting for other variables [41]. Another population-based study from South Africa found that HIV-infected persons had a lower prevalence of hypertension than HIV-uninfected persons $(19.5 \%$ vs $27.9 \%, p=0.001$ ) [42]. A longitudinal, prospective study in South Africa also found decreased risk of high blood pressure with HIV infection [43]. Yet, it has been well established that HIV infection has adverse effects on cardiovascular health [44]. The suggestion of an inverse association between HIV and hypertension should be viewed with caution and further evaluated.

In our prior pilot campaign in Kakyerere parish in 2011, we reported a higher prevalence of hypertension (28\%), which included self-reported hypertension. In this study we used a more rigorous definition of hypertension (using lowest of three BP measures or current use of antihypertensives) and did not include self-reported hypertension, which likely accounts for the difference in prevalence estimates between the two studies. Further, many participants reported to be hypertensive in our pilot study in 2011 were subsequently found not to have high BP on repeat screening at local health facilities. This over-reporting from our pilot study urged us to develop the more rigorous definition of hypertension that was implemented in our study.

Limitations of our study included incomplete attendance in the $\mathrm{CHC}$, as community members who did not attend the $\mathrm{CHC}$ were not screened for hypertension. Conducting a community-wide census allowed us to identify individuals who did not participate and account for bias resulting from differential participation by age in our prevalence estimate. We believe that our ageadjusted prevalence should be a reasonable estimate of the "true" prevalence, considering that age is the most significant risk factor for hypertension in this community. Measurement error in BP can lead to misclassification of hypertensive status; however, we attempted to minimize random error by taking three BP measurements, which increases the validity of our prevalence estimate.

The use of a multi-disease $\mathrm{CHC}$ to integrate services for HIV and other NCDs could be a promising public health approach in sub-Saharan Africa. Competition for limited funds and health care resources between NCDs and infectious diseases has already created a sensitive political issue [8-10]. Our approach avoids costs related to establishing new infrastructure and builds efficiency by using HIV as a platform to develop services for other chronic diseases. HIV is also the first chronic disease that has been successfully treated with high-quality, longterm care in resource-limited settings. Building alliances between HIV care and NCD services will improve and expand care for NCDs, consistent with recent declarations of the UN General Assembly [45].

\section{Conclusions}

There is a significant burden of hypertension in rural Uganda, which is comparable to that in developed nations. Among hypertensive patients, awareness of their condition is poor. And among those diagnosed, therapy rates are extremely low. Prevention efforts should focus on persons most likely to be hypertensive, such as overweight/ obese, alcohol consumers, and diabetics. Leveraging community-based HIV testing programs to incorporate NCD services is an efficient use of scarce health care resources in sub-Saharan Africa.

\section{Abbreviations \\ NCD: Non-communicable disease; HIV: Human immunodeficiency virus; CHC: Community health campaign; BP: Blood pressure; WHO: World Health Organization; ISH: International Society of Hypertension; JNC7: Seventh report of the Joint National Committee on prevention, detection, evaluation, and treatment of high blood pressure; BMl: Body mass index.}

\section{Competing interests}

The authors declare that they have no competing interests.

\section{Authors' contributions}

Conception, design and data collection: PK, DK, TDC, JK, EHG, VJ, GC, MLP, $H T, M R K, E D C, D V H$. Data analysis: PK, EHG EDC, DVH. Wrote and edited manuscript: PK, DK, TDC, JK, EHG, VJ, GC, MLP, HT, MRK, EDC, DVH.

All authors read and approved the final manuscript.

\section{Acknowledgements}

The authors would like to thank residents and local leaders of Kakyerere Parish for their tremendous support and participation in our study. We also wish to thank staff from the Bwizibwera Health Center IV and Mulago Joint Aids Program (MJAP) for their assistance. We thank the Ugandan Ministry of Health for the donation of HIV rapid test kits for use in the community health campaigns. Finally, we thank the Clinical and Translational Research Fellowship Program at UCSF for supporting PK's time. 


\section{Author details}

'HIV/AIDS Division, Department of Medicine, San Francisco General Hospital, University of California San Francisco, 995 Potrero Avenue, UCSF Box 0874 San Francisco, California 94110, USA. ${ }^{2}$ Makerere University-University of California San Francisco (MU-UCSF) Research Collaboration, Mbarara, Uganda. ${ }^{3}$ Mulago Joint AIDS Program, Kampala and Mbarara, Mbarara, Uganda. ${ }^{4}$ School of Public Health, University of California, Berkeley, California, USA. ${ }^{5}$ Gillings School of Global Public Health, University of North Carolina at Chapel Hill, Chapel Hill, North Carolina, USA. ${ }^{6}$ Department of Medicine, School of Medicine, Makerere University College of Health Sciences, Kampala, Uganda. ' Center for AIDS Prevention Studies, Department of Medicine, University of California San Francisco, San Francisco, California, USA.

Received: 1 July 2013 Accepted: 5 December 2013

Published: 9 December 2013

\section{References}

1. Leon DA: Cities, urbanization and health. Int J Epidemiol 2008, 37:4-8

2. Popkin BM: Global nutrition dynamics: the world is shifting rapidly toward a diet linked with noncommunicable diseases. Am J Clin Nutr 2006, 84:289-298.

3. Lawes CM, Vander Hoorn S, Rodgers A: Global burden of blood-pressurerelated disease, 2001. Lancet 2008, 371:1513-1518.

4. Yach D, Hawkes C, Gould CL, Hofman KJ: The global burden of chronic diseases: overcoming impediments to prevention and control. Jama 2004, 291:2616-2622.

5. Lim SS, Vos T, Flaxman AD, Danaei G, Shibuya K, Adair-Rohani H, Amann M Anderson HR, Andrews KG, Aryee M, et al: A comparative risk assessment of burden of disease and injury attributable to 67 risk factors and risk factor clusters in 21 regions, 1990-2010: a systematic analysis for the Global Burden of Disease Study 2010. Lancet 2012, 380:2224-2260.

6. Kearney PM, Whelton M, Reynolds K, Muntner P, Whelton PK, He J: Global burden of hypertension: analysis of worldwide data. Lancet 2005, 365:217-223.

7. Twagirumukiza M, De Bacquer D, Kips JG, de Backer G, Stichele RV, Van Bortel LM: Current and projected prevalence of arterial hypertension in sub-Saharan Africa by sex, age and habitat: an estimate from population studies. J Hypertens 2011, 29:1243-1252.

8. Mensah GA: Epidemiology of stroke and high blood pressure in Africa. Heart 2008, 94:697-705.

9. Opie $L H$, Seedat YK: Hypertension in sub-Saharan African populations. Circulation 2005, 112:3562-3568.

10. Smart T: HIV and non-communicable diseases (NCDs). In Book HIV and Non-communicable Diseases (NCDs). City: NAM; 2011.

11. Addo J, Smeeth L, Leon DA: Hypertension in sub-saharan Africa: a systematic review. Hypertension 2007, 50:1012-1018.

12. Maher D, Waswa L, Baisley K, Karabarinde A, Unwin N: Epidemiology of hypertension in low-income countries: a cross-sectional populationbased survey in rural Uganda. J Hypertens 2011, 29:1061-1068

13. Bloom DE, Cafiero ET, Jané-Llopis E, Abrahams-Gessel S, Bloom LR, Fathima S, Feigl AB, Gaziano T, Mowafi M, Pandya A, Prettner K, Rosenberg L, Seligman B, Stein AZ, Weinstein C: The global economic burden of noncommunicable diseases. In Book The Global Economic Burden of Noncommunicable Diseases (Editor ed.^eds.). City: World Economic Forum; 2011

14. Dalal S, Beunza JJ, Volmink J, Adebamowo C, Bajunirwe F, Njelekela M, Mozaffarian D, Fawzi W, Willett W, Adami HO, Holmes MD: Noncommunicable diseases in sub-Saharan Africa: what we know now. Int Epidemiol 2011, 40:885-901.

15. de Ramirez SS, Enquobahrie DA, Nyadzi G, Mjungu D, Magombo F, Ramirez M, Sachs SE, Willett W: Prevalence and correlates of hypertension: a cross-sectional study among rural populations in sub-Saharan Africa. J Hum Hypertens 2010, 24:786-795.

16. Hendriks ME, Wit FW, Roos MT, Brewster LM, Akande TM, de Beer IH Mfinanga SG, Kahwa AM, Gatongi P, Van Rooy G, et al: Hypertension in sub-Saharan Africa: cross-sectional surveys in four rural and urban communities. PLoS One 2012, 7:e32638.

17. Tumwesigye E, Wana G, Kasasa S, Muganzi E, Nuwaha F: High uptake of home-based, district-wide, HIV counseling and testing in Uganda. AIDS Patient Care STDS 2010, 24:735-741.
18. Lugada E, Millar D, Haskew J, Grabowsky M, Garg N, Vestergaard M, Kahn JG, Muraguri N, Mermin J: Rapid implementation of an integrated large-scale HIV counseling and testing, malaria, and diarrhea prevention campaign in rural Kenya. PloS one 2010, 5:e12435.

19. Rabkin M, El-Sadr WM: Why reinvent the wheel? Leveraging the lessons of HIV scale-up to confront non-communicable diseases. Glob Public Health 2011, 6:247-256.

20. Beaglehole R, Epping-Jordan J, Patel V, Chopra M, Ebrahim S, Kidd M, Haines A: Improving the prevention and management of chronic disease in low-income and middle-income countries: a priority for primary health care. Lancet 2008, 372:940-949.

21. Tollman SM, Kahn K, Sartorius B, Collinson MA, Clark SJ, Garenne ML: Implications of mortality transition for primary health care in rural South Africa: a population-based surveillance study. Lancet 2008, 372:893-901.

22. Rabkin M, Nishtar S: Scaling up chronic care systems: leveraging HIV programs to support noncommunicable disease services. J Acquir Immune Defic Syndr 2011, 57(Suppl 2):S87-S90.

23. Chamie G, Kwarisiima D, Clark TD, Kabami J, Jain V, Geng E, Petersen ML, Thirumurthy H, Kamya MR, Havlir DV, Charlebois ED: Leveraging rapid community-based HIV testing campaigns for non-communicable diseases in rural Uganda. PloS one 2012, 7:e43400

24. Whitworth JA: 2003 World Health Organization (WHO)/International Society of Hypertension (ISH) statement on management of hypertension.J Hypertens 2003, 21:1983-1992.

25. Chobanian AV, Bakris GL, Black HR, Cushman WC, Green LA, Izzo JL Jr, Jones DW, Materson BJ, Oparil S, Wright JT Jr, Roccella EJ: Seventh report of the joint national committee on prevention, detection, evaluation, and treatment of high blood pressure. Hypertension 2003, 42:1206-1252.

26. Uganda Clinical Guidelines. http://www.health.go.ug/docs/ucg_2010.pdf.

27. Labhardt ND, Balo JR, Ndam M, Manga E, Stoll B: Improved retention rates with low-cost interventions in hypertension and diabetes management in a rural African environment of nurse-led care: a cluster-randomised trial. Trop Med Int Health 2011, 16:1276-1284.

28. Klein RJ, Schoenborn CA: Age adjustment using the, projected U.S population. Healthy People 2010 Stat Notes 2000, 2001:1-10

29. Ahmad OB, Boschi-Pinto C, Lopez AD, Murray CJL, Lozano R, Inoue M: Age standardization of rates: a new WHO standard. In Book Age Standardization of Rates: A New WHO standard (Editor ed.^eds.). Geneva: World Health Organization; 2001.

30. Syed FF, Sani MU: Recent advances in HIV-associated cardiovascular diseases in Africa. Heart 2013, 99:1146-1153.

31. Musinguzi G, Nuwaha F: Prevalence, awareness and control of hypertension in Uganda. PloS one 2013, 8:e62236.

32. Twagirumukiza M, Van Bortel LM: Management of hypertension at the community level in sub-Saharan Africa (SSA): towards a rational use of available resources. J Hum Hypertens 2011, 25:47-56.

33. Unwin N, Setel P, Rashid S, Mugusi F, Mbanya JC, Kitange H, Hayes L, Edwards R, Aspray T, Alberti KG: Noncommunicable diseases in subSaharan Africa: where do they feature in the health research agenda? Bull World Health Organ 2001, 79:947-953.

34. Ezzati M, Lopez AD, Rodgers A, Vander Hoorn S, Murray CJ: Selected major risk factors and global and regional burden of disease. Lancet 2002, 360:1347-1360.

35. Murray CJ, Lopez AD: Global mortality, disability, and the contribution of risk factors: Global Burden of Disease Study. Lancet 1997, 349:1436-1442

36. Fezeu L, Kengne AP, Balkau B, Awah PK, Mbanya JC: Ten-year change in blood pressure levels and prevalence of hypertension in urban and rural Cameroon. J Epidemiol Community Health 2010, 64:360-365.

37. Cappuccio FP, Micah FB, Emmett L, Kerry SM, Antwi S, Martin-Peprah R, Phillips RO, Plange-Rhule J, Eastwood JB: Prevalence, detection, management, and control of hypertension in Ashanti, West Africa. Hypertension 2004, 43:1017-1022

38. van der Sande MA, Milligan PJ, Nyan OA, Rowley JT, Banya WA, Ceesay SM, Dolmans WM, Thien T, McAdam KP, Walraven GE: Blood pressure patterns and cardiovascular risk factors in rural and urban gambian communities. J Hum Hypertens 2000, 14:489-496.

39. Edwards R, Unwin N, Mugusi F, Whiting D, Rashid S, Kissima J, Aspray TJ, Alberti KG: Hypertension prevalence and care in an urban and rural area of Tanzania. J Hypertens 2000, 18:145-152. 
40. Wamala JF, Karyabakabo Z, Ndungutse D, Guwatudde D: Prevalence factors associated with hypertension in Rukungiri district, Uganda-a community-based study. Afr Health Sci 2009, 9:153-160.

41. Barnighausen T, Welz T, Hosegood V, Batzing-Feigenbaum J, Tanser F, Herbst K, Hill C, Newell ML: Hiding in the shadows of the HIV epidemic: obesity and hypertension in a rural population with very high HIV prevalence in South Africa. J Hum Hypertens 2008, 22:236-239.

42. Malaza A, Mossong J, Barnighausen T, Newell ML: Hypertension and obesity in adults living in a high HIV prevalence rural area in South Africa. Plos one 2012, 7:e47761.

43. Schutte AE, Schutte R, Huisman HW, van Rooyen JM, Fourie CM, Malan NT, Malan L, Mels CM, Smith W, Moss SJ, et al: Are behavioural risk factors to be blamed for the conversion from optimal blood pressure to hypertensive status in Black South Africans? A 5-year prospective study. Int J Epidemiol 2012, 41:1114-1123.

44. Grinspoon SK: Metabolic syndrome and cardiovascular disease in patients with human immunodeficiency virus. Am J Med 2005, 118(Suppl 2):23S-28S.

45. Political Declaration of the High-level Meeting of the General Assembly on the Prevention and Control of Non-communicable Diseases. http://www.who.int/ nmh/events/un_ncd_summit2011/political_declaration_en.pdf.

doi:10.1186/1471-2458-13-1151

Cite this article as: Kotwani et al: Epidemiology and awareness of hypertension in a rural Ugandan community: a cross-sectional study. BMC Public Health 2013 13:1151.

\section{Submit your next manuscript to BioMed Central and take full advantage of:}

- Convenient online submission

- Thorough peer review

- No space constraints or color figure charges

- Immediate publication on acceptance

- Inclusion in PubMed, CAS, Scopus and Google Scholar

- Research which is freely available for redistribution 